\title{
Regularization of the Coulomb scattering problem
}

\author{
V. G. Baryshevskii, I. D. Feranchuk, and P. B. Kats \\ Byelorussian State University, 4, F. Skariny Av., 220050, Minsk, Republic of Belarus
}

(Received 10 March 2004; published 5 November 2004)

\begin{abstract}
The exact solution of the Schrödinger equation for the Coulomb potential is used within the scope of both stationary and time-dependent scattering theories in order to find the parameters which determine the regularization of the Rutherford cross section when the scattering angle tends to zero but the distance $r$ from the center remains finite. The angular distribution of the particles scattered in the Coulomb field is studied on rather a large but finite distance $r$ from the center. It is shown that the standard asymptotic representation of the wave functions is inapplicable in the case when small scattering angles are considered. The unitary property of the scattering matrix is analyzed and the "optical" theorem for this case is discussed. The total and transport cross sections for scattering the particle by the Coulomb center proved to be finite values and are calculated in the analytical form. It is shown that the effects under consideration can be important for the observed characteristics of the transport processes in semiconductors which are determined by the electron and hole scattering by the field of charged impurity centers.
\end{abstract}

DOI: $10.1103 /$ PhysRevA.70.052701

PACS number(s): 03.65.Nk

\section{INTRODUCTION}

The scattering of nonrelativistic charged particles by the Coulomb center is one of the canonical problems both in classical and quantum mechanics which is known as the Rutherford problem. It is a generally accepted view that the differential cross section $d \sigma(\theta)$ of the particle scattering to the solid angle $d \Omega$ is of the same form in both cases (for example, Refs. [1,2])

$$
d \sigma(\theta)=\sigma(\theta) d \Omega=\left(\frac{\alpha}{2 m v^{2}}\right)^{2} \frac{d \Omega}{\sin ^{4} \theta / 2},
$$

where $m$ and $v$ are the particle mass and velocity, respectively, with parameter $\alpha$ defining the amplitude of the Coulomb potential $U(r)=\alpha / r$.

Thus, the main measurable characteristic of the scattering process in the Coulomb field has the nonintegrable singularity in the limit $\theta \rightarrow 0$ (in quantum theory the singularity exists also in the scattering amplitude). Fortunately, this singularity does not lead to any problem when describing most of real experiments because particles are scattered by the systems with the total zero charge. In this case the singularities due to the scattering centers of opposite signs are compensated and the cross section proves to be regular in the entire angular range. There are, however, some physical systems where one should solve the problem of regularization when calculating such integral scattering characteristics as the total $\sigma_{t o t}$ and transport $\sigma_{t r}$ cross sections

$$
\sigma_{t o t}=\int d \sigma(\theta), \quad \sigma_{t r}=\int(1-\cos \theta) d \sigma(\theta) .
$$

For example, one can mention the calculation of kinetic process characteristics in plasma and impurity semiconductors or collisions of charged particles in beams. In such cases one should introduce some phenomenological parameter $\theta_{\min }$ for cutting off the cross section (1) with angles $\theta<\theta_{\text {min }}$. This parameter can be defined by various physical reasons. Particularly, in the framework of classical mechanics a small scattering angle is determined by particles with a large impact parameter [3] connected with a long range character of the Coulomb potential. Therefore, a small angle cone can be excluded from the consideration because of a finite transversal width $a$ of the incident beam with $\theta_{\text {min }} \sim a / r$ [4].

Other approaches are used when the mobility of charge carriers is calculated in impurity semiconductors. At present Brooks-Herring [5] and Conwell-Weisskopf [6] models are most commonly used for this problem. These models correspond to different ways of estimating the parameter $\theta_{\min }$ connected with screening the Coulomb potential. However, such estimations are only of qualitative character and some additional phenomenological parameter should be introduced for a more precise description of the mobility as it has been recently shown in the paper [7]. Accurate calculations of the integral values characterizing the charge carrier scattering by impurities is important because of high accuracy of measuring these values in real semiconductors (for example Ref. [8]) Solution of this problem is of great interest also for analysing the electron transport in nanostructures such as quantum wires [9], superlattices and films [10], nanotubes [11].

Regularization problem for the Coulomb cross section becomes more vital in the framework of quantum theory. The matter is that wave functions for the states of the continuous spectrum are accurately known [1]; they have no singularities, even in the case of a plane incident wave which corresponds to the beam with an infinite transversal width. This means that the singularity of the scattering amplitude is not an intrinsic feature of the Coulomb system within the scope of a quantum mechanical description. It might be due to a rather incorrect description and interpretation of the asymptotic behavior of the wave function in this case. So, there should exist some characteristic, or "kinematic," regularization parameter $\theta_{0}$ which unlike the value $\theta_{\min }$ is not connected with the initial state of the system. In a general case the regularized cross section should depend on both parameters. 
It is critical to emphasize that some specific characteristics of the Coulomb scattering problem have been widely discussed in monographs and textbooks. For example, it is shown in book [4] that the connection between the impact parameter and the scattering angle becomes indefinite in the case of $\theta=0$, therefore the scattering cross section for zero angle cannot be calculated in terms of classical dynamics. It is also well known that a long-range character of the Coulomb potential leads to a logarithmic distortion of the phase in the asymptotic form of the wave function [1]. However, the problem of the cross-section regularization has not been considered in these works.

This problem was first analyzed in our paper [12]. It was shown that the standard asymptotic representation of the wave function was not actually formed at small angles when considering the scattering processes by long-range potentials $\left[U(r) \sim 1 / r^{s} ; s \leqslant 3\right]$. As a result the canonical definition of the scattering amplitude proved to be inapplicable. In our work [12] Born approximation over the potential $U(r)$ and the time-dependent collision theory [13] were used in order to calculate the scattering cross section without any singularities. We can also mention a number of papers ([14] and references therein) where it was shown that the interference between incident and scattered waves changed the asymptotic form of the wave function and could be significant under real experimental conditions even in the case of some short-range potentials.

In the present paper we consider the nonasymptotic analysis of the observed characteristics for the nonrelativistic Coulomb scattering problem outside the framework of the perturbation theory. We use the exact solutions of the Schrödinger equation in order to answer the following questions: (1) which "intrinsic" kinematic parameter determines the regularization of the Rutherford cross section in the framework of the stationary scattering theory; (2) how does this regularization depend on such "external" parameters as the transversal width of the incident wave packet or effective "cutting off" of the potential; (3) how can one calculate nonasymptotic values for the integral scattering characteristics $\sigma_{t o t}, \sigma_{t r} ;$; (4) what is the analogue of the "optical" theorem $4 \pi \operatorname{Im} f(0)=\kappa \sigma_{\text {tot }}$ in the case of the Coulomb potential $[1,2]$ ? It seems to us that the answers to these questions have an important methodical value for understanding the scattering processes in the field of long-range potentials but have not been discussed before. Besides, these results can also be essential for some applications such as the above-mentioned transport processes in semiconductors with charged impurities.

The paper is organized as follows. In Sec. II the differential scattering cross section is determined without an asymptotic representation of the wave function and the kinematic regularization parameter is found for the Rutherford problem. In Sec. III the most important integral characteristics of the scattering problem are calculated. In Sec. IV the scattering operator and the conservation of the total flux are analyzed. The time-dependent consideration of the collision process is discussed in Sec. V and the influence of the incident beam parameters and the potential screening on the observed scattering characteristics is studied. The scattering characteristics of the carriers in nondegenerated semiconduc-

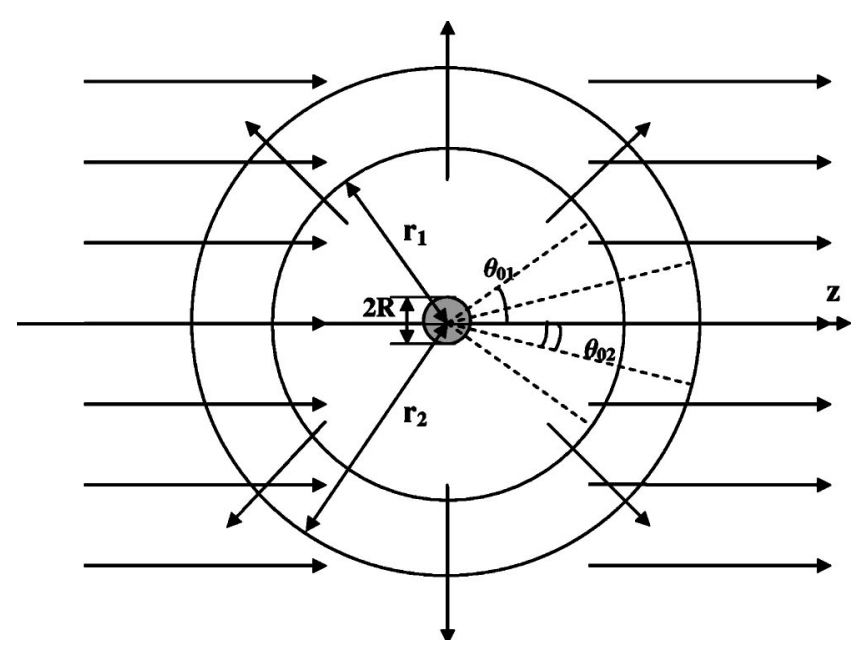

FIG. 1. Sketch of the scattering process in the stationary case.

tors with charged impurities are calculated in Sec. VI, and the results are compared with the experimental values of the carrier mobility in real systems.

\section{NONASYMPTOTIC CALCULATION OF THE DIFFERENTIAL CROSS SECTION FOR THE COULOMB SCATTERING}

Let us remember the main definitions of the scattering theory in terms of stationary quantum mechanics. It is well known [1] that in the general case one should find the wave function of the continuous spectrum $\psi_{\vec{k}}(\vec{r})$ as the solutions of the Schrödinger equation

$$
\left[-\frac{\hbar^{2}}{2 m}\left(\Delta+k^{2}\right)+U(\vec{r})\right] \psi_{k}(\vec{r})=0,
$$

under the following asymptotic boundary conditions (Fig. 1 shows all the necessary notations):

$$
\begin{gathered}
\psi_{\vec{k}}(\vec{r}) \sim e^{i \vec{k} \cdot \vec{r}}, \quad \vec{k} \cdot \vec{r} \rightarrow-\infty, \\
\psi_{\vec{k}}(\vec{r}) \sim e^{i \vec{k} \cdot \vec{r}}+f(\theta) \frac{e^{i k r}}{r}, \quad r \gg R .
\end{gathered}
$$

Here $\vec{k}$ is the wave vector; the value $R$ determines a characteristic radius of the potential action with the center at $r$ $=0(R \rightarrow \infty$ in the case of the Coulomb field $)$. The wave function is supposed to be normalized to one particle, so the flux density in the incident state is

$$
\begin{aligned}
\vec{j}= & \frac{\hbar}{2 i m}\left[\psi_{k}(\vec{r})^{*} \vec{\nabla} \psi_{k}(\vec{r})-\psi_{k}(\vec{r}) \vec{\nabla} \psi_{k}(\vec{r})^{*}\right] \simeq \frac{\hbar}{m} \vec{k} \equiv \vec{j}_{0}, \\
& \vec{k} \cdot \vec{r} \rightarrow-\infty .
\end{aligned}
$$

The flux density in the asymptotic state (5) is divided into two components:

$$
\vec{j}=j_{l} \frac{\vec{k}}{k}+j_{s c} \frac{\vec{r}}{r}, \quad r \gg R
$$


One of them $j_{l}$ (a longitudinal component) corresponds to the particles passed through the field without any interaction and the second one $j_{s c}$ (a radial component) describes the scattered particles. This results in the standard definition of the cross section:

$$
\begin{gathered}
d \sigma(\theta)=\frac{j_{s c}}{j_{0}} r^{2} d \Omega, \\
\sigma(\theta)=|f(\theta)|^{2} .
\end{gathered}
$$

It should be noted that the longitudinal flux also changes $j_{l}<j_{0}$, its decrease being determined by the total scattering cross section in accordance with the "optical" theorem [1].

It is evident that definition (8) is based on the asymptotic mode (5) for the wave function at the observation point $r$. According to the terminology used in radio-physics and optics (for example [15]), it means that the particle should leave the "near" zone, the potential action is still considerable, and pass to the "far," or "wave," zone. The boundary between these zones is conditioned by the fact that the interference between incident and scattered waves becomes negligible, so the difference between their phases satisfies the inequality

$$
\begin{gathered}
k r-\vec{k} \cdot \vec{r}=2 k r \sin ^{2}(\theta / 2)>1, \\
\theta>\theta_{0} \equiv \sqrt{\frac{2}{k r}} .
\end{gathered}
$$

We suppose further that for all real collisions the condition $k r \gg 1$ is fulfilled.

It is clear that the boundary of the "wave" zone depends both on the distance $r$ from the center and the scattering angle $\theta$ (Fig. 1). It means that in the general case there is some part of the particle flux which cannot be described by the asymptotic wave function (5) even for rather a large distance $r$. This property certainly does not depend on the radius of the potential action. However, the question arises: what is the contribution of these particles to the integral scattering process? When the distance from the center $r$ is fixed, the number of particles scattered to the "near" zone $\theta<\theta_{0}$ can be estimated as

$$
N_{\text {dif }} \simeq j_{0} \sigma(0) \theta_{0}^{2} \sim \frac{j_{0} \sigma(0)}{k r} .
$$

The cross section $\sigma(0)$ is restricted for the potentials with a finite action radius $R$, therefore $N_{\text {dif }}$ decreases quickly at a large distance. It means that the contribution of these particles to the observed scattering characteristics is negligible for most real experiments. The detailed analysis of the "near" and "wave" zone formation for the scattering problem with a short-range potential has been recently considered in paper [14].

The situation changes fundamentally in the case of a longrange potential $(R \rightarrow \infty)$. The value $N_{d i f}$ can even increase with the distance and its contribution to the formation of the scattering flux can be significant. Particularly, a similar estimation in the case of the Coulomb field is of the form

$$
N_{d i f} \gg j_{0}\left(\frac{\alpha}{m v^{2}}\right)^{2} \frac{4}{\theta_{0}^{2}} \sim k r .
$$

It means that the asymptotic boundary condition (5) is not applicable in the entire range of the scattering angles and the nonasymptotic expression for the wave function should be used in the case of small angles. It is important to stress that this circumstance is not related to the width of an incident beam and is conditioned by the characteristic feature of the potential itself.

So, the considered regularization problem for the Rutherford cross section within the scope of the stationary scattering theory is reduced to the analysis of the space flux distribution in terms of the well known exact solution of Eq. (3) with the potential $U(r)=Z e^{2} / r$ but without turning to the asymptotic representation of the wave function.

We will use the following form of the normalized wave function [1]

$$
\begin{gathered}
\psi_{k}(\vec{r})=N e^{i \vec{k} \cdot \vec{r}} F( \pm i \xi, 1, i(k r-\vec{k} \cdot \vec{r})), \quad N=e^{ \pm(\pi / 2) \xi} \Gamma(1 \mp i \xi), \\
\xi=\frac{\alpha}{\hbar v}, \quad \alpha=Z e^{2}, \quad v=\frac{\hbar \kappa}{m},
\end{gathered}
$$

where $F(a, b, t)$ is the confluent hypergeometric function; $\Gamma(t)$ is the gamma function; the upper sign in the formulas corresponding to the attraction field and the lower one corresponds to the repulsion potential.

Let us show that the flux density in the formula (6) calculated with the exact wave function can also be divided into two components according to formula (7) as it was done in the asymptotic mode. For this purpose one can use the representation of the function $F$ as the superposition of two confluent hypergeometric functions of the third genus $U_{1,2}(a, b, t)[16]$

$$
\begin{gathered}
F( \pm i \xi, 1, i z)=\frac{1}{\Gamma( \pm i \xi)} U_{1}( \pm i \xi, 1, i z)+\frac{1}{\Gamma(1 \mp i \xi)} U_{2}( \pm i \xi, 1, i z), \\
U_{1}( \pm i \xi, 1, i z)=(z)^{ \pm i \xi} \frac{e^{i z}}{\Gamma(1 \mp i \xi)} e^{\mp \pi \xi} G_{1}( \pm i \xi, i z), \\
G_{1}( \pm i \xi, i z)=\int_{0}^{\infty} e^{-u} u^{\mp i \xi}\left(1-\frac{u}{i z}\right)^{ \pm i \xi} \frac{d u}{i z-u}, \\
U_{2}( \pm i \xi, 1, i z)=(z)^{\mp \pi \xi} \frac{1}{\Gamma( \pm i \xi)} e^{\mp \pi \xi} G_{2}( \pm i \xi, i z), \\
G_{2}( \pm i \xi, i z)=\int_{0}^{\infty} e^{-u} u^{ \pm i \xi-1}\left(1+\frac{u}{i z}\right)^{\mp i \xi} d u, \\
z=(k r-\vec{k} \vec{r})=k r(1-\cos \theta) .
\end{gathered}
$$

Let us also mention the relation between these functions and the confluent hypergeometric functions of the second genus $U(a, b, t)[21]$

$$
U_{1}(a, b, t)=U(b-a, b,-t) e^{t} e^{ \pm i \pi(a-b)},
$$




$$
\begin{gathered}
U_{2}(a, b, t)=U(a, b, t) e^{ \pm i a \pi}, \\
U(a, b, t)=\frac{1}{\Gamma(a)} \int_{0}^{\infty} e^{-t u} u^{a-1}(1+u)^{b-a-1} d u .
\end{gathered}
$$

When the Rutherford cross section is calculated by means of the standard definition this representation enables one to find the asymptotic form of the wave function within the limit $z \gg 1$ [1]. This case corresponds to the "wave" zone when the function $U_{1}$ transforms to the spherical wave and the function $U_{2}$ tends to the plane wave. However, both these functions are also well defined in the "near" zone $(z<1)$ when they can be calculated by means of the following series [16]:

$$
\begin{aligned}
& U_{1}( \pm i \xi, 1, i z)=\frac{1}{2} \Gamma( \pm i \xi)\{F( \pm i \xi, 1, i z) \\
& +\frac{e^{\mp 2 \pi \xi}-1}{2 \pi i}([2 \ln (i z) \mp i \pi \operatorname{coth}(\pi \xi)-i \pi \\
& +2 \psi( \pm i \xi)] F( \pm i \xi, 1, i z) \\
& +2 \sum_{m=1}^{\infty} \frac{\Gamma(m \pm i \xi)}{\Gamma( \pm i \xi)(m !)^{2}}[\psi(m \pm i \xi)-\psi( \pm i \xi) \\
& \left.\left.+2 \psi(1)-2 \psi(m+1)](i z)^{m}\right)\right\} \\
& U_{2}( \pm i \xi, 1, i z)=\frac{1}{2} \Gamma(1 \mp i \xi)\{F( \pm i \xi, 1, i z) \\
& -\frac{e^{\mp 2 \pi \xi}-1}{2 \pi i}([2 \ln (i z) \mp i \pi \operatorname{coth}(\pi \xi)-i \pi \\
& +2 \psi( \pm i \xi)] F( \pm i \xi, 1, i z) \\
& +2 \sum_{m=1}^{\infty} \frac{\Gamma(m \pm i \xi)}{\Gamma( \pm i \xi)(m !)^{2}}[\psi(m \pm i \xi)-\psi( \pm i \xi) \\
& \left.\left.+2 \psi(1)-2 \psi(m+1)](i z)^{m}\right)\right\},
\end{aligned}
$$

where $\psi(t)$ is the logarithmic derivative of the gamma function.

When representation (13) is used in formula (6) one should take into account only the derivatives of the exponents because the conditions $k r \gg 1$; $z \sim 1$ are supposed to be fulfilled. For example,

$$
\begin{aligned}
-i\left(\frac{\vec{r}}{r} \cdot \vec{\nabla}\right)\left[e^{i k r} G_{1}(i z)\right] & =e^{i k r}\left[k G_{1}+\frac{z}{r} G_{1}^{\prime}(i z)\right] \\
& \simeq e^{i k r} k G_{1}\left[1+O\left(\frac{1}{k r}\right)\right] .
\end{aligned}
$$

This estimation is evident for the "near" and "intermediate" zones of the scattering angles $\left(\theta \leqslant \theta_{0}, z \leqslant 1\right)$. On the other hand, in the "far" zone $\left(\theta \gg \theta_{0}, z \gg 1\right)$ the following estimation can be found using the asymptotic form of the function $G_{1}[1]$ :

$$
\left|G_{1}^{\prime}(i z) \simeq \frac{1}{z}\right| G_{1}(i z) \mid .
$$

Therefore approximation (16) can be used in the entire range of the scattering angles.

This representation permits one to find the scattering flux $j_{s c}$ directed to the observation point along the vector $\vec{r}$ without using the asymptotic form (5) of the wave function. As a result the scattering cross section can be estimated in the entire range of the angles $\theta$ in the following way:

$$
\begin{aligned}
\sigma_{1}(\theta) d \theta & =\sin \theta r^{2} \int_{0}^{2 \pi} d \varphi \frac{j_{s c}}{j_{0}} d \theta \\
& =2 \xi \sinh (\pi \xi) e^{\mp \pi \xi}\left|G_{1}( \pm i \xi, \iota z)\right|^{2} r^{2} \sin \theta d \theta
\end{aligned}
$$

One can see that the differential cross section $\sigma_{1}(\theta)$ is finite for any angles in spite of the fact that the function $G_{1}( \pm i \xi, i z)$ has a logarithmic singularity at a zero angle as it follows from Eq. (15). But there is a nontrivial dependence of this value on the distance between the center and the observation point because of a long-range action of the potential on the particle. The result of this action at small angles ("near" zone) is not confined to varying the phase of the scattering amplitude as it happens for the asymptotic range of angles ("wave" zone) [1].

If one considers the behavior of the function $G_{1}( \pm i \xi, i z)$ in relation to the scattering angle, the "kinematic" parameter $\theta_{0}$ for regularizing the Rutherford cross section can be naturally introduced. The asymptotic range of angles corresponding to the "wave" zone is actually defined by the condition

$$
\begin{gathered}
z=k r-\vec{k} \cdot \vec{r} \simeq \frac{1}{2} k r \theta^{2} \gg 1, \\
\theta \gg \theta_{0}=\sqrt{\frac{2}{k r}} \ll 1, \quad x \gg 1, \\
x=\frac{\theta}{\theta_{0}}, \quad z \simeq x^{2} .
\end{gathered}
$$

Here a dimensionless value $x$ is introduced as a convenient variable for the angles comparable with the width of the "near" zone. Certainly, in the range of $x \gg 1$ the standard asymptotic representation of the integral in the definition of the function $G_{1}( \pm i \xi, i z)$ results in formula (1) with a new variable

$$
\sigma_{1}(\theta) \simeq 2 \pi\left(\frac{\xi}{k}\right)^{2} \frac{\sqrt{2}(k r)^{3 / 2}}{x^{3}}=8 \pi\left(\frac{\alpha}{m v^{2}}\right)^{2} \frac{1}{\theta^{3}}, \quad x \gg 1 .
$$

It is well known that the interference between the scattering flux and the flux directed along the initial velocity of the particle is not taken into account within the scope of any quantum scattering theory based on the solutions of the sta- 

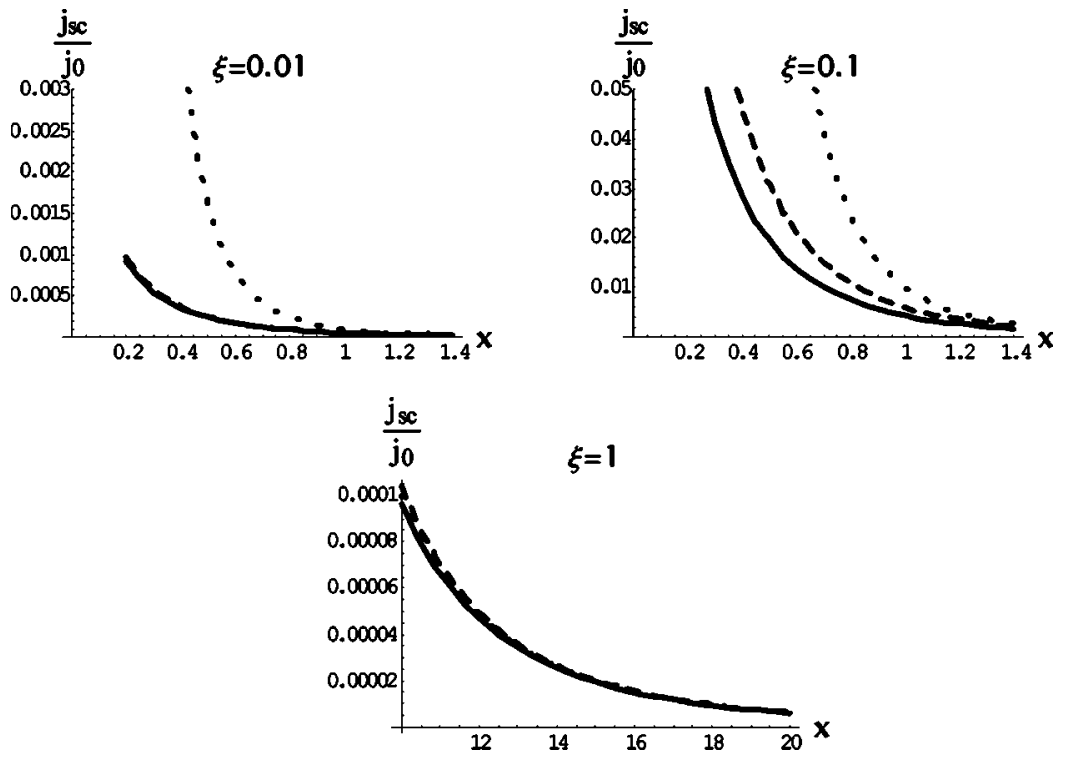

tionary Schrödinger equation [1]. So, in order to use formula (17) in the range of $x<1$ corresponding to the "near" zone, one should compare it with the angle width of the zone where the above mentioned interference is still valid. It is clear that the angle width of such an "interference" zone does not depend on the dynamics of the interaction between the particle and the field. It is defined only by a transversal width $a$ of the incident particle wave packet. With this transversal width of the wave packet, the incident particles have the angle divergence $\theta_{\text {int }}$ conditioned by the uncertainty relation which can be estimated as follows:

$$
\theta_{i n t} \simeq \frac{1}{k a}
$$

It means that one can distinguish the scattering flux from the incident one if the angular width of the "near" zone is more than the uncertainty relation (20):

$$
\theta_{\text {int }}<\theta<\theta_{0}=\sqrt{\frac{2}{k r}}, \quad \frac{k a^{2}}{r}>1, \quad r<k a^{2} .
$$

We will see below (Sec. IV) that these inequalities are fulfilled for the distance $r$, when one can neglect by the spread of the wave packet. This condition is usually implied in the time-dependent theory of collisions [13]. A detailed analysis of the fluxes will also be considered in Sec. IV, but now one can estimate the contribution of the "interference" zone to the integral scattering characteristics which in our case unlike the asymptotic analysis are finite values. Therefore, the ratio of the particle fluxes scattered into the "interference" and "near" zones can be estimated as

$$
\begin{aligned}
\delta & =\frac{j_{i n t}}{j_{d i f}} \simeq \int_{0}^{\theta_{i n t}} \sigma_{1}(\theta) d \theta / \int_{0}^{\theta_{0}} \sigma_{1}(\theta) d \theta \simeq\left(\frac{\theta_{\text {int }}}{\theta_{0}}\right)^{2} \simeq \frac{2 r}{k a^{2}} \\
& \ll 1 .
\end{aligned}
$$

It remains small under standard conditions of the collision theory [13], and one can analyze the distribution of the flux density in the "near" zone neglecting its interference with the incident flux. It permits one to find the leading terms of the differential scattering cross section at small angles using series (15)

$$
\sigma_{1}(\theta) \simeq 8 \sqrt{2} \xi e^{\mp \pi \xi} \sinh (\pi \xi) x(\ln x)^{2} \frac{r^{3 / 2}}{\sqrt{k}} .
$$

Figure 2 compares the accurate and asymptotic scattered (Rutherford) fluxes for different values of the variable $x$ and the parameter $\xi$. It must be kept in mind that the behavior of the nonasymptotic flux in the "near" zone for scattering by attractive and repulsive centers is quite different to that of the Rutherford cross section (1) which is independent of the potential sign for any value of $\xi$. One can see that the regularized differential cross section (17) in the "near" zone is, in fact, noninvariant relative to the sign of the charge if the parameter $\xi \geqslant 1$. It should be noted that the effect of a slightly different interaction of the charge carriers with the impurities of different signs is well known in the semiconductor physics. It is usually analyzed in terms of the Friedel sum rule [18] based on the partial expansion of the scattering amplitude in the series of orbital momenta.

As it follows from Eq. (23), the scattering flux in the case of the attractive potential varies rather slowly with the increase of the parameter $\xi$, but it increases exponentially in the case of the repulsion. Such behavior of the cross section takes place only over a narrow angle domain (21) and compensates the exponential decrease of the flux along the line $\theta=0$ which is well known for the repulsive potential [1].

\section{INTEGRAL CHARACTERISTICS FOR THE COULOMB SCATTERING PROBLEM}

Let us now calculate the integral scattering characteristics for the problem under consideration. According to Eq. (17) the nonasymptotic expression for the total cross section is determined by the following integral: 

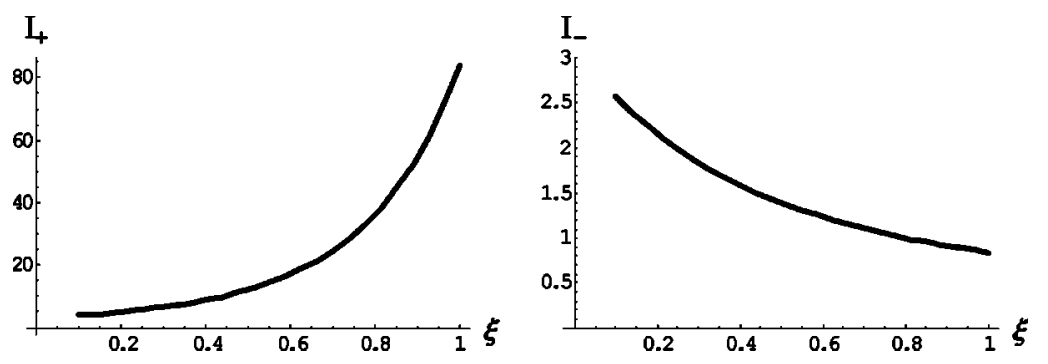

FIG. 3. Universal functions $I_{ \pm}(\xi)$ which define the total scattering cross section as the function of the parameter $\xi$ for the cases of repulsion and attraction.

$$
\sigma_{t o t}=\int_{0}^{\pi} 2 \xi \sinh (\pi \xi) e^{\mp \pi \xi}\left|G_{1}( \pm i \xi, i z)\right|^{2} r^{2} \sin \theta d \theta
$$

One can use a new variable $z$ in this integral

$$
\sigma_{\text {tot }}=\int_{0}^{2 k r} \xi \sinh (\pi \xi) e^{\mp \pi \xi}\left|G_{1}( \pm i \xi, i z)\right|^{2} \frac{2 r}{k} d z
$$

and represent it as the sum of two integrals

$$
\begin{aligned}
\sigma_{\text {tot }}= & \frac{2 r}{k} \xi \sinh (\pi \xi) e^{\mp \pi \xi}\left\{\int_{0}^{\infty}\left|G_{1}( \pm i \xi, i z)\right|^{2} d z\right. \\
& \left.-\int_{2 k r}^{\infty}\left|G_{1}( \pm i \xi, i z)\right|^{2} d z\right\} .
\end{aligned}
$$

In the second integral the asymptotic representation for the function $G_{1}$ (13) can be used over the whole integration interval

$$
G_{1}( \pm i \xi, i z) \simeq \frac{e^{ \pm(\pi / 2) \xi} \Gamma(1 \mp i \xi)}{i z}
$$

thus leading to the following simple result:

$$
I_{2}=\frac{2 r}{k} \xi e^{\mp \pi \xi} \sinh (\pi \xi) \int_{2 k r}^{\infty}\left|G_{1}( \pm i \xi, i z)\right|^{2} d z \simeq \frac{\pi \xi^{2}}{k^{2}} .
$$

The order of this integral being $(k r)^{-1}$ in comparison with the first integral, its contribution to the total cross section can be neglected. This permits one to find how the value $\sigma_{\text {tot }}$ depends on the most essential parameters of the problem

$$
\begin{gathered}
\sigma_{t o t}=\frac{2 \pi r}{k} \xi^{2} I_{ \pm}(\xi), \\
I_{ \pm}(\xi)=e^{\mp \pi \xi} \int_{0}^{\infty}\left|U_{1}(1 \pm i \xi, 1, i z)\right|^{2} d z .
\end{gathered}
$$

Here we again use the canonical form for the confluent hypergeometric function of the second genus [16], the universal functions $I_{ \pm}(\xi)$ depending only on the variable $\xi$. They are found by the converging integrals and can be easily calculated numerically. Figure 3 shows the results.

As distinct from the standard scattering theory for the short-range potentials, the regularized total cross section for the Coulomb field depends on the distance $r$ between the detector and scattering center. One can understand the physi- cal meaning of this result if one takes into account that according to the above analysis [see Eq. (21)] the distance is restricted by the inequality $r<k a^{2}$ which is actually fulfilled for real experimental conditions [13]. As a result the maximal value of the total cross section is

$$
\sigma_{\max }=2 \pi a^{2} \xi^{2} I_{ \pm}(\xi) .
$$

It means that the maximal cross section is proportional to the area of the transversal section of the incident particles beam. It is quite natural because of a long range character of the Coulomb potential when finally all the particles of the beam are scattered with the probability depending on the dimensionless parameter $\xi$. When the detector is situated a smaller distance $r$ from the center, it will register a fewer number of the scattered particles depending on $r$.

It is important to emphasize that the considered peculiarities of the Coulomb problem should be taken into account only for small angle scattering experiments for the potential without screening and the processes depending on the integral scattering characteristics. In other cases our results completely coincide with the standard Rutherford consideration.

Now let us consider another integral characteristic of the scattering process, namely, the transport cross section which is a very important quantity for a lot of applications. It is determined by the formula

$$
\sigma_{t r}=\int_{0}^{\pi} 2 \xi \sinh (\pi \xi) e^{\mp \pi \xi}\left|G_{1}( \pm i \xi, i z)\right|^{2} r^{2} \sin \theta(1-\cos \theta) d \theta
$$

If one uses the variable $z$ in this integral and comes back to the hypergeometric function of the second genus, Eq. (30) transforms itself as follows:

$$
\begin{gathered}
\sigma_{t r}=\frac{2 \pi \xi^{2}}{k^{2}} e^{\mp \pi \xi} A_{ \pm}^{t r}(\xi), \\
A_{ \pm}^{t r}(\xi)=\int_{0}^{2 k r}|U(1 \pm i \xi, 1, i z)|^{2} z d z .
\end{gathered}
$$

The integrand function for the transport cross section is essentially suppressed in the range of small angles in comparison with the total cross section. Therefore $\sigma_{t r}$ is determined only by the logarithm of the distance to the observation point whereas $\sigma_{\text {tot }}$ is proportional to this distance. Besides, this function decreases rather slowly for large $z$ and one cannot use the trick analogous to Eq. (26) for $\sigma_{t r}$ Nevertheless, a series of transformations of the integral $I_{ \pm}^{t r}(\xi)$ permits one to find the analytical dependence on the coordi- 

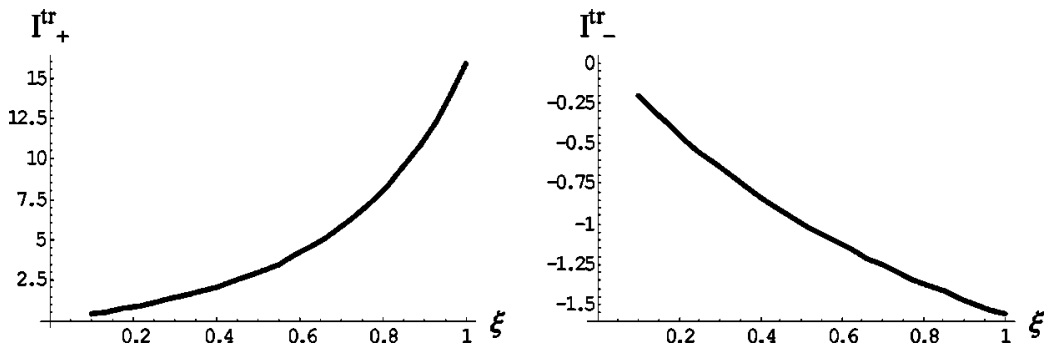

FIG. 4. Universal functions $I_{ \pm}^{t r}(\xi)$ for the transport cross section for the cases of attraction and repulsion. Solid line, the case of attraction; dashed line, the case of repulsion.

nate $r$ with the accuracy of the order $(k r)^{-1}$. Let us divide the integral into two parts in the following way:

$A_{ \pm}^{\operatorname{tr}}(\xi)=\int_{0}^{1}|U(1 \pm i \xi, 1, i z)|^{2} z d z+\int_{1}^{2 k r}|U(1 \mp i \xi, 1, i z)|^{2} z d z$

If one uses the asymptotic formula for the hypergeometric function [16]

$$
|U(1 \pm i \xi, 1, i z)|^{2} \simeq \frac{e^{ \pm \pi \xi}}{z^{2}}+O\left((z)^{-3}\right),
$$

the second term in this integral is identically transformed

$$
\int_{1}^{2 k r}\left[|U(1 \pm i \xi, 1, i z)|^{2}-\frac{e^{ \pm \pi \xi}}{z^{2}}\right] z d z+\int_{1}^{2 k r} \frac{e^{ \pm \pi \xi}}{z} d z
$$

Here the second integral is easily calculated analytically, but now the integrand expression in the first one decreases rather quickly and the estimation analogous to Eq. (26) can be used:

$$
\begin{aligned}
\int_{1}^{\infty} & {\left[|U(1 \pm i \xi, 1, i z)|^{2}-\frac{e^{ \pm \pi \xi}}{z^{2}}\right] z d z-\int_{2 k r}^{\infty}\left[|U(1 \pm i \xi, 1, i z)|^{2}\right.} \\
& \left.-\frac{e^{ \pm \pi \xi}}{z^{2}}\right] z d z \\
\simeq & \int_{1}^{\infty}\left[|U(1 \pm i \xi, 1, i z)|^{2}-\frac{e^{ \pm \pi \xi}}{z^{2}}\right] z d z \\
& +O\left[(k r)^{-1}\right] .
\end{aligned}
$$

As a result the transport cross section is defined by wellconverged integrals:

$$
\begin{aligned}
\sigma_{t r}= & \frac{2 \pi \xi^{2} e^{\mp} \pi \xi}{k^{2}}\left\{\int_{0}^{1}|U(1 \pm i \xi, 1, i z)|^{2} z d z\right. \\
& \left.+\int_{1}^{\infty}\left[|U(1 \pm i \xi, 1, i z)|^{2}-\frac{e^{ \pm \pi \xi}}{z^{2}}\right] z d z+e^{ \pm \pi \xi} \ln (2 k r)\right\} \\
= & \frac{2 \pi \xi^{2}}{k^{2}}\left(I_{ \pm}^{t r}(\xi)+\ln (2 k r)\right) \\
& \left.I_{ \pm}^{t r}(\xi)=e^{\mp}\right) \\
& +\int_{1}^{\infty}\left[\left.\left|\int_{0}^{1}\right| U(1 \pm i \xi, 1, i z)\right|^{2} z d z\right.
\end{aligned}
$$

Figure 4 shows the results of a numerical calculation of the universal functions $I_{ \pm}^{t r}(\xi)=\left[\left(k^{2} / 2 \pi \xi^{2}\right) \sigma_{t r}-\ln (2 k r)\right]$.

\section{SCATTERING OPERATOR AND CONSERVATION OF THE FLUX WITHIN SCOPE OF THE STATIONARY THEORY}

As it follows from the results of the preceding section the integral scattering characteristics calculated on the basis of a nonasymptotic consideration increase with the distance $r$ from the center to the observation point. At the first sight it seems that this can contradict the conservation of the particle total flux when $r$ becomes rather large. However, let us show that this dependence expresses only the fact that the potential influences the scattering process at any distance from the center but the scattering flux remains considerably less than the integral incident flux at any $r$. For a qualitative analysis one should take into account that within the scope of the stationary scattering theory the quantum state of the incident particle is described by a plane wave. Then the total incident flux $J_{0}$ through the sphere of radius $r$ corresponding to the observation point can be estimated as follows:

$$
J_{0} \simeq j_{0} \pi r^{2} \text {. }
$$

Then the ratio of the scattering and incident integral fluxes is

$$
\frac{j_{s c}^{t o t}}{j_{0}} \simeq \frac{\sigma_{t o t}}{r^{2}}=\frac{2 \pi}{k r} \xi^{2} I_{ \pm} \ll 1 .
$$

It is also important to consider this problem more precisely. It is known [1] that in the quantum theory of scattering with short-range potentials the "optical" theorem is the consequence of the total flux conservation when the amplitude of scattering at a zero angle is a finite value. We use the same approach [1] in order to find the consequence of this condition for the case of a nonasymptotic analysis of the Coulomb scattering.

Let us represent the general solution of the Schrödinger equation for the case of the elastic scattering as the linear combination of the functions (12) with arbitrary coefficients $\Phi(\vec{n})$ which determine the probability amplitudes to find the state with the wave vector $\vec{k}=k \vec{n}$ in the initial packet:

$$
\begin{aligned}
\Psi(\vec{r}) & =\int \Phi(\vec{n}) \psi_{k}(\vec{r}) d \Omega_{\vec{n}} \\
& =N \int \Phi(\vec{n}) e^{i k r \vec{n} \cdot \vec{n}^{\prime}} F\left( \pm i \xi, 1, i k r\left(1-\vec{n} \cdot \vec{n}^{\prime}\right)\right) d \Omega_{\vec{n}} \\
& =\int \Phi(\vec{n})\left[(z)^{ \pm i \xi} \frac{e^{i k r}}{\Gamma( \pm i \xi)} G_{1}( \pm i \xi, i z)\right.
\end{aligned}
$$




$$
\begin{gathered}
\left.+(z) \mp i \xi \frac{e^{i k r \vec{n} \cdot \vec{n}^{\prime}}}{\Gamma( \pm i \xi)} G_{2}( \pm i \xi, i z)\right] d \Omega_{\vec{n}}, \\
\vec{n}^{\prime}=\frac{\vec{r}}{r}, \quad z=k r\left(1-\vec{n} \cdot \vec{n}^{\prime}\right),
\end{gathered}
$$

where $d \Omega_{\vec{n}}$ is the element of the solid angle in the direction of the vector $\vec{n}$.

In accordance with the physical interpretation of the contributions made by the functions $G_{1}, G_{2}$ to the total wave function (12), the first term in Eq. (35) describes that part of the integral scattering operator [1] which corresponds to the formation of the scattering wave. The term, proportional to the function $G_{2}$, describes the deformation of the wave packet conditioned by changing the plane wave in the Coulomb field. One can estimate the second term by the same method that was used for proving the "optical" theorem in the case of a short-range potential [1]. If the condition $k r$ $\gg 1$ is fulfilled, the main contributions to this integral are made by small intervals near the points of stationary phases when integrating over $\vec{n}$. These points correspond to the vectors $\vec{n}_{1}=-\vec{n}^{\prime}$ and $\vec{n}_{2}=\vec{n}^{\prime}$. Near the first point the variable $z$ $\simeq 2 k r$ is very large. Therefore one can use the asymptotic expression for the function $G_{2}( \pm i \xi, i z)$ and the integrand has no singularities in this case. As a result the contribution to the integral from the domain close to this point defines the converged spherical wave with a standard logarithmic distortion of its phase [1]

$$
\sim 2 \pi i \frac{e^{-i k r \mp i \xi \ln 2 k r}}{k r} \Phi(-\vec{n}) .
$$

When estimating the contribution to the integral from the second point of the stationary phase corresponding to the scattering at small angles one should take into account that the function $G_{2}( \pm i \xi, i z)$ has a logarithmic singularity at the point $z=0$. Nevertheless, rather a smooth weight function $\Phi(\vec{n})$ can be removed from the integral at the point $\vec{n}=\vec{n}^{\prime}$. It leads to the following estimation:

$$
\begin{aligned}
& \sim 2 \pi \Phi(\vec{n}) e^{\mp(\pi / 2) \xi} \frac{e^{i k r}}{k r \Gamma( \pm i \xi)}\left[\int_{0}^{\infty}(z)^{\mp i \xi} e^{-i z} G_{2}( \pm i \xi, i z) d z\right. \\
& \left.-\int_{2 k r}^{\infty}(z)^{\mp i \xi} e^{-i z} G_{2}( \pm i \xi, i z) d z\right] .
\end{aligned}
$$

The second integral in this expression can be omitted within the limit $k r \gg 1$ and the initial wave function is represented in the form:

$$
\begin{aligned}
\Psi(\vec{r}) \simeq & 2 \pi i \frac{e^{-i k r \mp i \xi \ln 2 k r}}{k r} \Phi\left(-\vec{n}^{\prime}\right)-2 \pi i \frac{e^{i k r}}{k r}\left[A \Phi\left(\vec{n}^{\prime}\right)\right. \\
& \left.+\int \hat{f}\left(\vec{n}, \vec{n}^{\prime}\right) \Phi(\vec{n}) d \vec{n}\right], \\
A & =\frac{i e^{\mp(\pi / 2) \xi}}{\Gamma( \pm i \xi)} \int_{0}^{\infty}(z)^{\mp i \xi} e^{-i z} G_{2}( \pm i \xi, i z) d z,
\end{aligned}
$$

$$
\begin{aligned}
\hat{f}\left(\vec{n}, \vec{n}^{\prime}\right)= & \frac{i k r}{2 \pi \Gamma( \pm i \xi)} e^{\mp(\pi / 2) \xi} e^{ \pm i \xi \ln k r}(1 \\
& \left.-\vec{n} \cdot \vec{n}^{\prime}\right)^{ \pm i \xi} G_{1}\left[ \pm i \xi, i k r\left(1-\vec{n} \cdot \vec{n}^{\prime}\right)\right] .
\end{aligned}
$$

It is more convenient to rewrite this expression in terms of the hypergeometric function of the second genus

$$
\begin{gathered}
A=i e^{\mp(\pi / 2) \xi} \int_{0}^{\infty} e^{-i z} U( \pm i \xi, 1, i z) d z, \\
\hat{f}\left(\vec{n}, \vec{n}^{\prime}\right)=-\frac{i k r \Gamma(1 \mp i \xi)}{2 \pi \Gamma( \pm i \xi)} e^{\mp(\pi / 2) \xi} U\left(1 \mp i \xi, 1,-i k r\left(1-\vec{n} \cdot \vec{n}^{\prime}\right)\right) .
\end{gathered}
$$

Now the function is represented as the superposition of the incoming and outgoing spherical waves and this permits one to introduce the scattering matrix [1] as the following integral operator:

$$
\hat{S}\left(\vec{n}, \vec{n}^{\prime}\right) \simeq A \delta_{\vec{n}, \vec{n}^{\prime}}+\hat{f}\left(\vec{n}, \vec{n}^{\prime}\right) .
$$

Here $\delta_{\vec{n}, \vec{n}^{\prime}}$ is the unit operator which corresponds to the wave passed without any scattering and the parameter $A$ defines the change in its amplitude (in the case of a short-range potential $A=1[1])$. The integral over the angles from the scattering operator $\int \hat{f}^{*}\left(\vec{n}, \vec{n}^{\prime}\right) \hat{f}\left(\vec{n}^{\prime}, \vec{n}\right) d \vec{n}^{\prime}$ exactly coincides with the expression for the total cross section (28), multiplied by $k^{2} / 4 \pi^{2}$. A long-range character of the potential is reflected in the fact that the scattering matrix elements depend on the coordinate $r$ due to the distortion of the front of a spherical wave. However, it is very important to introduce this operator because it defines the kernel of the collision integral in the kinetic equations describing various transport processes [20]. But if one uses such operator in the collision integral for one-particle distribution function the additional averaging over the coordinate should be fulfilled. The dependence of the function $f\left(\vec{n}^{\prime}, \vec{n}\right)$ on the coordinate is rather smooth, therefore the value $r$ in this function can be substituted as an average distance between the scattering centers, if the correlation between these centers can be neglected (see below Sec. VI). An analogous substitution was used in some generally accepted models for the regularization of the transport cross section of the scattering by charged impurities in semiconductors $[5,6]$.

The unitary property of the matrix $\hat{S}\left(\vec{n}, \vec{n}^{\prime}\right)$ leads to the "optical" theorem for short-range potentials [1]. But if one uses this condition for the Coulomb potential, because the operator $\hat{f}\left(\vec{n}, \vec{n}^{\prime}\right)$ has a logarithmic singularity at coinciding arguments one should define the method for calculating the integral from the product of singular functions $\hat{f}\left(\vec{n}, \vec{n}^{\prime}\right)$ and $\delta_{\vec{n}, \vec{n}^{\prime}}$ in the operator $\hat{S} \hat{S}^{+}$. It means actually that the asymptotic estimation of the integral in Eq. (36) is unacceptable for the operator which is quadratic over the scattering matrix. Therefore, let us analyze separately the flux conservation considering the following integral:

$$
I=\int d V[\vec{\nabla} \cdot \vec{j}(\vec{r})] \equiv \int[\vec{r} \cdot \vec{j}(\vec{r})] r d \Omega_{\vec{n}},
$$




$$
\vec{j}(\vec{r})=\frac{\hbar}{2 m i}\left\{\Psi^{*}(\vec{r}) \vec{\nabla} \Psi(\vec{r})-\Psi(\vec{r}) \vec{\nabla} \Psi^{*}(\vec{r})\right\},
$$

with the total wave function (35).

Using the superposition (35) in formula (39) one can take into account the completeness of the coefficients $\Phi(\vec{n})$. Then the integration along all directions in this integral is equivalent to the integral from the flux $\vec{j}_{s t}$ calculated by means of a general formula (39) but with stationary wave functions $\psi_{k}(\vec{r})$ defined by Eq. (12) (for definiteness, let us consider the attractive potential)

$$
\begin{gathered}
\vec{j}_{s t}(\vec{r})=\frac{\hbar \pi \xi e^{\pi \xi}}{m \sinh \pi \xi}\left\{\vec{k}|F(i \xi, 1, i(k r-\vec{k} \cdot \vec{r}))|^{2}\right. \\
\left.-\xi\left(\vec{k}-k^{\frac{\vec{r}}{r}}\right) \operatorname{Im}\left(F F_{1}^{*}\right)\right\}, \\
\vec{\nabla} F(i \xi, 1, i(k r-\vec{k} \cdot \vec{r}))=\xi\left(\vec{k}-k^{\frac{\vec{r}}{r}}\right) F(i \xi+1,2, i(k r-\vec{k} \cdot \vec{r})) \\
\equiv \xi\left(\vec{k}-k_{\frac{r}{r}}^{\vec{r}}\right) F_{1} .
\end{gathered}
$$

Generally speaking, the value $I$ is equal to zero identically because of the flux conservation for the stationary scattering problem. The "optical" theorem follows from this condition if an asymptotic form (5) for the wave function can be used [1]. But in the considered problem this condition means that the flux directed along the vector $\vec{k}$ (it determines the change in the intensity of the incident wave), and the scattering flux along the vector $\vec{r}$ are related as follows:

$$
\begin{gathered}
\int d \Omega_{\vec{n}}(\vec{k} \cdot \vec{n})|F(i \xi, 1, i(k r-\vec{k} \cdot \vec{r}))|^{2} \\
=\xi \int d \Omega_{\vec{n}}(\vec{k} \cdot \vec{n}-k) \operatorname{Im}\left(F F_{1}^{*}\right) .
\end{gathered}
$$

As it is shown above, the integrals over the angles for the Coulomb scattering problem include an essential contribution defined by the "near" zone. Therefore, both parts of Eq. (41) depend on the coordinate $r$ and the standard asymptotic expressions for the "optical" theorem are unacceptable because the total cross section and the scattering amplitude at zero angle tend to infinity in this case. But if one shows that the leading terms of Eq. (41) are equal within the limit of large $r(k r \gg 1)$ it can be considered as the analog of the "optical" theorem for the Coulomb potential.

In order to prove this let us use a new variable for the integrals in Eq. (41)

$$
z=k r-\vec{k} \cdot \vec{r}, \quad \sin \theta d \theta=\frac{d z}{k r},
$$

and transform them as follows:

$$
\begin{aligned}
\int_{0}^{2 k r}|F(i \xi, 1, i z)|^{2} d z= & \int_{0}^{2 k r} \frac{z}{k r}\left\{|F|^{2}+\xi \operatorname{Im}[F(-i \xi, 1,-i z) F(i \xi\right. \\
& +1,2, i z)]\} d z
\end{aligned}
$$

One can estimate the integrals from the confluent hypergeometric functions within the limit $k r \gg 1$ by means of the following approach. The integral on the left side of Eq. (42) can be identically transformed

$$
\begin{aligned}
J_{1}= & \int_{0}^{2 k r}|F(i \xi, 1, i z)|^{2} d z=\lim _{\delta \rightarrow 0}\left[\int_{0}^{\infty}|F|^{2} e^{-\delta z} d z\right. \\
& \left.-\int_{2 k r}^{\infty}|F|^{2} e^{-\delta z} d z\right] .
\end{aligned}
$$

The parameter $\delta \rightarrow 0$ is introduced for the regularization of both integrals at an upper limit. The asymptotic form of the function $F$ can be used in the second term and the first term can be expressed by the hypergeometric function $F(\alpha, \beta, \gamma, z)$ using the formula (see, for example [1])

$$
\begin{aligned}
J(\lambda)= & \int_{0}^{\infty} e^{-\lambda z} z^{\gamma-1} F(\alpha, \gamma, k z) F\left(\alpha^{\prime}, \gamma, k^{\prime} z\right) d z \\
= & \Gamma(\gamma) \lambda^{\alpha+\alpha^{\prime}-\gamma}(\lambda-k)^{-\alpha}\left(\lambda-k^{\prime}\right)^{-\alpha^{\prime}} \\
& \times F\left[\alpha, \alpha^{\prime}, \gamma, \frac{k k^{\prime}}{(\lambda-k)\left(\lambda-k^{\prime}\right)}\right] .
\end{aligned}
$$

When the integrals from the functions with different second arguments are calculated, the following recursion relation can be used [16]:

$$
F(\alpha+1, \gamma+1, z)=\frac{\gamma}{z}[F(\alpha+1, \gamma, z)-F(\alpha, \gamma, z)] .
$$

Let us give also the leading terms of the asymptotic expansions for the functions $F$ and $F_{1}$ which are used for calculating the integrals with the limits $(2 k r, \infty)$

$$
\begin{aligned}
F(i \xi, 1, i z) \simeq & e^{-\pi \xi / 2}\left[\frac{z^{-i \xi}}{\Gamma(1-i \xi)}\left(1-\frac{i \xi^{2}}{z}+\frac{\xi^{2}(1+i \xi)^{2}}{2 z^{2}}\right)\right. \\
- & \left.-\frac{i z^{i \xi} e^{i z}}{z \Gamma(i \xi)}\right], \\
F_{1}=F(i \xi+1,2, i z) \simeq & \frac{i e^{-\pi \xi / 2}}{z}\left[\frac{z^{-i \xi}}{\Gamma(1-i \xi)}\left(1-\frac{\xi(1+i \xi)}{z}\right)\right. \\
& \left.-\frac{z^{i \xi} e^{i z}}{\Gamma(1+i \xi)}\left(1-\frac{\xi(1-i \xi)}{z}\right)\right] .
\end{aligned}
$$

As a result the leading term on the left side of Eq. (42) is in the form

$$
\begin{aligned}
J_{1} \simeq & e^{-\pi \xi} \frac{\sinh \pi \xi}{\pi \xi}\left\{2 k r+2 \xi-\frac{1}{k r}\left[\xi^{2}\right.\right. \\
& \left.\left.+\operatorname{Re}\left(\frac{\Gamma(1+i \xi) e^{-2 i k r-2 i \xi \ln 2 k r}}{\Gamma(-i \xi)}\right)\right]\right\}+O\left(\frac{1}{(k r)^{2}}\right) .
\end{aligned}
$$

This value determines the variation of the flux directed along the incident wave vector and it increases linearly with the distance from the scattering center similarly to the total cross section. As mentinoed above [Eq. (40)], this increase is 
not connected with the increase of the particle flux but only describes the distorted part of the wave front which extends together with $r$ because of a long-range character of the potential.

Calculating the integral

$$
J_{2}=\frac{1}{k r} \int_{0}^{2 k r}|F(i \xi, 1, i z)|^{2} z d z
$$

by a similar method leads to the following result:

$$
\begin{aligned}
J_{2} \simeq & e^{-\pi \xi} \frac{\sinh \pi \xi}{\pi \xi}\left\{2 k r+\frac{2}{k r}\left[\xi ^ { 2 } \left(-\frac{3}{2}-\operatorname{Re} \psi(1+i \xi)\right.\right.\right. \\
& \left.\left.+\ln 2 k r)-\operatorname{Re}\left(\frac{\Gamma(1+i \xi) e^{-2 i k r-2 i \xi \ln 2 k r}}{\Gamma(-i \xi)}\right)\right]\right\} \\
& +O\left[\frac{1}{(k r)^{2}}\right],
\end{aligned}
$$

where $\psi(x)$ is the logarithmic derivative of $\Gamma$ function [16].

The last integral in Eq. (42)

$$
\begin{aligned}
J_{3} & =\frac{\xi}{k r} \int_{0}^{2 k r} z \operatorname{Im}[F(-i \xi, 1,-i z) F(i \xi+1,2, i z)] d z \\
& =-\frac{\xi}{k r} \int_{0}^{2 k r} \operatorname{Re}[F(-i \xi, 1,-i z) F(i \xi+1,1, i z)] d z,
\end{aligned}
$$

transforms itself as follows:

$$
\begin{aligned}
J_{3} \simeq & \frac{2}{k r} e^{-\pi \xi} \frac{\sinh \pi \xi}{\pi \xi}\left\{\xi^{2}[1+\operatorname{Re} \psi(1+i \xi)-\ln 2 k r]\right. \\
& \left.+\frac{1}{2} \operatorname{Re}\left(\frac{\Gamma(1+i \xi) e^{-2 i k r-2 i \xi \ln 2 k r}}{\Gamma(-i \xi)}\right)+\xi k r\right\}+O\left[\frac{1}{(k r)^{2}}\right] .
\end{aligned}
$$

Substituting Eqs. (45)-(48) for Eq. (42) shows that the latter is satisfied with the considered accuracy. Besides, one can see that the left side of Eq. (42) corresponds to the total cross section (28), and the right side of Eq. (42) corresponds to the imaginary part of the scattering operator (38) with $\vec{n}$ $=\vec{n}^{\prime}$. So, one can consider this calculation as the proof of the "optical" theorem for the Coulomb scattering problem.

\section{THE WAVE PACKET PROPAGATION IN THE COULOMB FIELD}

As it follows from the results of the preceding sections, the regularization of the Rutherford cross section is determined by the characteristic angle

$$
\theta_{0}=\sqrt{\frac{2}{k r}}
$$

which corresponds to the boundary of the "near" zone and is consider as the kinematic parameter (KP) of the system. However, in real scattering experiments the incident particle is actually represented by the localized wave packet [13]. Besides, the Coulomb potential is screened at some distance
$R_{s}$, depending on the properties of the medium where the collision occurs. Therefore, in the general case the problem is characterized by some additional parameters that can be considered as external parameters (EP). So, one should estimate the conditions under which KP are more important for the cross section regularization than EP. One will take into account the two most essential EP: the screening angle $\theta_{s}$ and the incident angle parameter $\theta_{i n t}$, depending on the wave packet transversal width $a$ and defining the interference zone between the incident and scattered waves (see also Sec. II). A simple estimation of these parameters leads to

$$
\theta_{s}=\frac{1}{k R_{s}}, \quad \theta_{\text {int }}=\frac{1}{k a} .
$$

The kinematic regularization is evidently most essential if the angle width of the "near" zone is larger than the characteristic angle intervals connected with EP, i.e., the following conditions are fulfilled

$$
\theta_{0}>\theta_{s}, \quad \frac{k R_{s}^{2}}{r}>1, \quad \theta_{0}>\theta_{i n t}, \quad \frac{k a^{2}}{r}>1 .
$$

The first inequality depends on the screening mechanism and should be analyzed for each concrete system as it will be shown below (Sec. VI) for the scattering by impurities in semiconductors. In order to take into account the finite size of the wave packet in the second inequality in Eq. (51) one should use the time-dependent theory of collisions $[13,12]$, which will be considered in this section.

Let us suppose that the initial state of the particle at the moment $t=0$ is defined by the wave packet in the following form:

$$
\begin{gathered}
\Psi_{\vec{k}}(\vec{r}, 0)=\int d \vec{q} \Phi(\vec{q}-\vec{k}) e^{i \vec{q} \cdot\left(\vec{r}-\vec{r}_{0}\right)} \equiv e^{i \vec{k} \cdot\left(\vec{r}-\vec{r}_{0}\right)} G\left(\left|\vec{r}-\vec{r}_{0}\right|\right), \\
G(\rho)=\int d \vec{p} \Phi(\vec{p}) e^{i \vec{p} \cdot \vec{p}},
\end{gathered}
$$

where $\vec{r}_{0}$ is the coordinate corresponding to the initial position of the wave packet; $\Phi(\vec{p})$ are the probability amplitudes of the wave vector distribution near the center $\vec{k}$ in the initial state; $G(\rho)$ is the function which describes the form of the localized wave packet in the coordinate space [13].

In order to describe the evolution of the wave packet (52) it should be expanded in the solutions of the stationary Schrödinger equation with the Coulomb potential [12] (for definiteness let us consider the attractive potential)

$$
\psi_{\vec{k}}(\vec{r})=N e^{i \vec{k} \cdot \vec{r}} F\left[i \xi_{k}, 1, i(k r-\vec{k} \cdot \vec{r})\right] .
$$

In standard scattering experiments (Fig. 5) the initial position of the wave packet corresponds to the condition $z_{0}$ $\rightarrow-\infty$. In this case the stationary wave function $\psi_{k}(\vec{r})$ coincides with the plane wave [13] and the expansion of $\Psi_{k}(\vec{r}, 0)$ in the functions $\psi_{k}(\vec{r})$ includes the same coefficients as in the formula (52), with the accuracy of the terms being of the order $\left|z_{0}\right|^{-1}$, due to the logarithmic distortion of the wave 


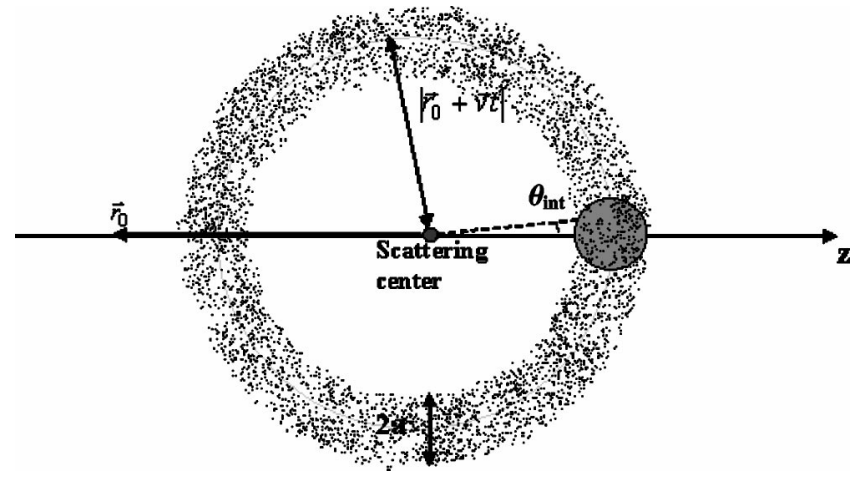

FIG. 5. Sketch of distribution of the probability density corresponding to the wave packet (56) at some moment $t$.

front in the Coulomb field [1]. As a result the wave function describing the wave packet state of an arbitrary moment of time is of the following form:

$$
\begin{aligned}
\Psi_{\vec{k}}(\vec{r}, t)= & \int d \vec{q} \Phi(\vec{q}-\vec{k}) e^{i \vec{q} \cdot \vec{r}} e^{-i \vec{q} \cdot \vec{r}_{0}} e^{\pi \xi_{q} / 2} \Gamma\left(1-i \xi_{q}\right) F\left(i \xi_{q}, 1, i(q r\right. \\
& -\vec{q} \cdot \vec{r})) e^{-i \hbar q^{2} t / 2 m} .
\end{aligned}
$$

As it is thoroughly analyzed in the monograph [13] the wave packet extension (diffraction) can be neglected during the interaction in real scattering experiments. This corresponds to the following approximation in the integrand expression in formula (53):

$$
\begin{gathered}
\vec{q}-\vec{k}=\vec{p}, \quad p \ll k, \quad \frac{\hbar q^{2}}{2 m} \simeq \frac{\hbar k^{2}}{2 m}+(\vec{p} \cdot \vec{v}), \quad \xi_{q} \simeq \xi=\frac{\alpha}{\hbar v}, \\
q \simeq k+\frac{(\vec{p} \cdot \vec{k})}{k},
\end{gathered}
$$

where $\vec{v}=\hbar \vec{k} / m$ is the group velocity of the center of the wave packet coinciding with the velocity of classical particles.

Let us briefly remember the results of the time-dependent collision theory for the case of a short-range potential when the asymptotic form (5) of the stationary wave function can be used for analyzing the wave packet evolution [13]

$$
\Psi_{\vec{k}}(\vec{r}, t)=\int d \vec{q} \Phi(\vec{q}-\vec{k}) e^{i \vec{q} \cdot \overrightarrow{r_{0}}}\left[e^{-i \vec{q} \cdot \vec{r}}+f\left(\theta_{q}\right) \frac{e^{i q r}}{r}\right] e^{-i \hbar q^{2} t / 2 m},
$$

where $\theta_{q}$ is the angle between the vectors $\vec{q}$ and $q \vec{r} / r$.

Now one can use expansions (54) and find the following result for the function $\Psi_{\vec{k}}(\vec{r}, t)$

$$
\begin{aligned}
\Psi_{k}(\vec{r}, t) \simeq & \left\{e^{i \vec{k} \cdot \vec{r}} G\left(\left|\vec{r}-\vec{r}_{0}-\vec{v} t\right|\right)+f\left(\theta_{k}\right) \frac{e^{i k r}}{r} G\left(\mid \begin{array}{r}
\vec{k} \\
k
\end{array}-\vec{r}_{0}\right.\right. \\
& -\vec{v} t \mid)\} e^{-i \vec{k} \cdot \vec{r}_{0}} e^{-i \hbar k^{2} t / 2 m} .
\end{aligned}
$$

Figure 5 shows schematically the distribution of the probability density corresponding to the wave packet (56) at some moment $t$. It demonstrates two essential results which actually represent the basis for using the quantum mechanical stationary scattering theory for describing the collisions between real particles [13]. First, the overlapping of the fluxes corresponding to the incident [the first term in formula (56)] and scattering particles should be taken into account if they are indistinguishable because of the uncertainty correlation for the incident wave packet $\theta_{i n t}=1 / \mathrm{ka}$. It means that the width $\theta_{0}$ of the "near" zone should be greater than this value. Besides, the scattering flux is localized in the spherical layer with the average radius $r \simeq\left|\vec{r}_{0}+\vec{v} t\right|$ and width $\sim a$. The angular distribution of the scattering particle within the limits of this layer is fully determined by the scattering amplitude $f\left(\theta_{k}\right)$ calculated on the basis of the stationary theory.

The expansions (54) can be used in the integral (55) if the integrand has no singularities in the range of the variable variation. This condition is not satisfied for the asymptotic form (5) in the case of the Coulomb field because the Rutherford amplitude includes unintegrable singularity. Let us show, however, that the representation of the wave packet similar to formula (56) also holds for the Coulomb problem if the expansion (56) is made on the basis of nonasymptotic representation (13) for the confluent hypergeometric function:

$$
\begin{aligned}
\Psi_{k}(\vec{r}, t)= & \int d \vec{q} \Phi(\vec{q} \\
& -\vec{k}) e^{-i \vec{q} \cdot \vec{r}} e^{\mp(\pi / 2) \xi_{q}}\left[\left(z_{q}\right)^{i \xi_{q}} \frac{e^{i q r}}{\Gamma\left(i \xi_{q}\right)} G_{1}\left(i \xi_{q}, i z_{q}\right)\right. \\
& \left.+\left(z_{q}\right)^{-i \xi \xi q} \frac{e^{i \vec{q} \cdot \vec{r}}}{\Gamma\left(i \xi_{q}\right)} G_{2}\left(i \xi_{q}, i z_{q}\right)\right] e^{-i \hbar q^{2} t / 2 m}, \\
z_{q}= & q r-\vec{q} \cdot \vec{r} .
\end{aligned}
$$

The functions $G_{1,2}$ are rather smooth and integrable. One can use expansion (54) for their arguments if the following condition is satisfied within the region $z_{q} \leqslant 1$, the latter being of the most essential variation of these functions:

$$
\begin{gathered}
z_{k} \geqslant \vec{p} \cdot\left(\frac{\vec{k}}{r}-\vec{r}\right) \simeq \operatorname{pr} \theta_{p}, \\
\theta^{2} \simeq \frac{1}{k r} \geqslant\left(\frac{1}{k a}\right)^{2}, \quad \frac{k a^{2}}{r} \leqslant 1 .
\end{gathered}
$$

It coincides with the above mentioned estimation (51) made on the basis of the qualitative analysis. It should also be emphasized that the same inequality permits one to neglect the extension of the wave packet during the collision [13]:

$$
\theta^{2} \simeq \frac{1}{k r} \geqslant\left(\frac{a}{r}\right)^{2}, \quad \frac{k a^{2}}{r} \leqslant 1 .
$$

As a result the functions $G_{1,2}$ in formula (57) can be removed out of the integral with the arguments corresponding to the center of the wave packet and this leads to the expression 


$$
\begin{aligned}
& \Psi_{k}(\vec{r}, t)=\left[\left(z_{k}\right)^{i \xi_{k}} \frac{e^{i k r}}{\Gamma\left(i \xi_{k}\right)} G_{1}\left(i \xi_{k}, i z_{k}\right) G\left(\left|r_{k}^{-\vec{k}}-\vec{r}_{0}-\vec{v} t\right|\right)\right. \\
& +\left(z_{k}\right)^{-i \xi_{k}} \frac{e^{i \vec{k} \cdot \vec{r}}}{\Gamma\left(i \xi_{k}\right)} G_{2}\left(i \xi_{k}, i z_{k}\right) G\left(\mid \vec{r}-\vec{r}_{0}\right. \\
& -\vec{v} t \mid)] e^{\mp(\pi / 2) \xi_{k}} e^{i-\vec{k} \cdot \vec{r}_{0}} e^{-i \hbar k^{2} t / 2 m}, \quad z_{k}=k r-\vec{k} \cdot \vec{r} .
\end{aligned}
$$

It means that the scattering process in the Coulomb field can be considered on the basis of the stationary theory as it takes place in the case of a short-range potential. Besides, the incident and scattered wave packets are extending in space separately excluding an unessential domain of their overlapping.

\section{CALCULATION OF CHARGE CARRIER MOBILITY IN EXTRINSIC SEMICONDUCTORS}

It is important to consider a specific physical system where the described peculiarities of the scattering process in the Coulomb field can appear for some observed characteristics. According to the estimation (51), they are possible if the following inequality is fulfilled:

$$
\frac{k R_{s}^{2}}{r}>1 \text {. }
$$

Here $R_{s}$ is the screening radius of the Coulomb potential in a medium and it depends on the screening mechanism in the system. The value $r$ is defined by the distance between the scattering center and the detector or by the average distance between two subsequent collisions if the scattering operator (32) is used for describing of kinetic processes in the system.

In the present paper the nonasymptotic scattering theory will be used for analyzing of charge carrier mobility in extrinsic semiconductors at low temperature. In this case the concentration of the impurity centers determines both the type of the carriers and their concentration and also the main contribution to the resistance of the semiconductor [17]. The problem has been recently analyzed in detail in paper [7] and the results of various phenomenological models for regularization of the Rutherford cross section are compared with experimental data [7]. It is shown that the widely used models of Brooks-Herring [5], and Conwell-Weisskopf [6] do not completely describe the experimental dependence of the mobility on temperature and impurity concentration. The authors of paper [7] fitted the experimental data much better by means of an additional phenomenological parameter, having the physical meaning of the characteristic collision time. Such parameter seems to take into account partly the influence of the "near" zone (see Sec. II) on the formation of the scattered flux. So, the regularization of the scattering problem in the Coulomb field is of interest not only as the methodological problem but also as the applied one.

Let us consider an extrinsic semiconductor with the donor concentrations $n_{1}$ and acceptor $n_{2}$ in the charge states $Z_{1} e$ and $Z_{2} e$, respectively (in most real structures the impurities with the charge $\left|Z_{1,2}\right|=1$ are important), $e$ being the absolute value of the electron charge.

In general case the value $n_{e}$ is determined by both thermally excited carriers and carriers due to impurities. Semiconductors with a wide forbidden zone are analyzed in paper [7] and the value $n_{e}$ can be estimated as

$$
n_{e} \simeq Z_{1} n_{1}-Z_{2} n_{2}=n,
$$

for the considered low temperature.

Let us also introduce another parameter which is more common in semiconductor physics: $K$ is compensation and usually has quite a small value $K \simeq 0.1$ [7]

$$
n_{1}=\frac{n}{Z_{1}-K Z_{2}}, \quad n_{2}=\frac{n K}{Z_{1}-K Z_{2}}, \quad K=\frac{n_{2}}{n_{1}} .
$$

It is well known [17] that the Coulomb potential screening in semiconductors is defined by several factors. On the one hand, there is a static dielectric constant $\epsilon$ conditioned by the electrons from the valency band which does not change a long-range character of the potential. On the other hand, the Debye screening of the potential by free electrons (or holes) leads to its cut off at the distance [17]

$$
R_{s} \simeq \sqrt{\frac{\epsilon k_{B} T}{4 \pi e^{2} n_{e}}},
$$

where $k_{B}$ is Boltzmann constant; $T$ is the crystal temperature; $n_{e}$ is the concentration of free charge carriers (electrons in the conduction band for $n$-type semiconductors or holes in the valence band for $p$-type semiconductors).

The average distance $r$ between scattering centers and the characteristic were vector for the carriers in formula (62) can be estimated as

$$
r \simeq n^{-1 / 3}, \quad k=\frac{\sqrt{2 m^{*} E}}{\hbar} \simeq \frac{\sqrt{3 m^{*} k_{B} T}}{\hbar},
$$

with $m^{*}$ being the carrier effective mass.

As a result condition (62) leads to the following inequality:

$$
\frac{\left(3 \epsilon m^{*}\right)^{1 / 2}\left(k_{B} T\right)^{3 / 2}}{4 \pi e^{2} \hbar n^{2 / 3}}>1,
$$

which is fulfilled in the entire range of the density and temperature considered in [7].

In most applications the theoretical estimation of the carrier mobility is based on the approximation of the relaxation time $\tau$ and Maxwell's velocity distribution. It leads to the following formula (for definiteness $n$-type semiconductors are considered) [17]:

$$
\begin{gathered}
\mu=\frac{e}{m^{*}}\langle\tau\rangle, \\
\langle\tau\rangle=\left[\int_{0}^{\infty} E^{3 / 2} e^{-E / k_{B} T}\right]^{-1} \int_{0}^{\infty} \tau(E) E^{3 / 2} e^{-E / k_{B} T} .
\end{gathered}
$$

Here the relaxation time is supposed to be averaged on the energy of carriers with Maxwell's distribution. 
It is known [19] that if several scattering mechanisms take place (e.g., the scattering by donors and acceptors), to obtain a more accurate result the additional averaging on the types of scattering centers should be fulfilled:

$$
\begin{gathered}
\tau(E)=\frac{\tau_{1}(E) \tau_{2}(E)}{\tau_{1}(E)+\tau_{2}(E)}, \\
\tau_{1,2}(E)=\frac{1}{n_{1,2} v \sigma_{t r 1,2}},
\end{gathered}
$$

where the indexes 1,2 correspond to the scattering by donors and acceptors; $\sigma_{t r 1,2}$ is the transport cross section for the cases of attraction and repulsion. In accordance with Sec. III these values are defined by the formulas

$$
\sigma_{t r 1,2}=\frac{2 \pi \xi_{1,2}^{2} e^{\mp} \pi \xi_{1,2}}{k^{2}} \int_{0}^{2 k r_{1,2}}\left|U\left(1 \pm i \xi_{1,2}, 1, i z\right)\right|^{2} z d z
$$

The more accurate formula than in Eq. (33) is used here because in this case the condition $k r \gg 1$ cannot be fulfilled.

The interaction parameters between carriers and scattering centers in the considered cases are the following:

$$
\xi_{1,2}(E)=\frac{Z_{1,2} e^{2}}{\epsilon \hbar v}
$$

and the static dielectric constant of the crystal is taken into account.

According to formulas (69) the transport cross section depends on the potential charge as distinct from its calculation with the Rutherford cross section. A similar effect ("phase shift") is well known for extrinsic semiconductors and is considered usually by means of the Fridel sum rule [18]. An indefinite parameter $r$ is included in Eq. (69). If the value $\mu$ is calculated in the totally microscopic way it should be averaged on the space distribution of the impurities in the sample. It is equivalent to the integration of expression (66) by $r$ taking into account Eq. (69). However, the transport cross section has a smooth logarithmic behavior at $r$ which can be substituted in Eq. (69) as the average distance between the impurities with the considered accuracy. Then the value $r=0.5 n_{i}^{-1 / 3}$ can be used in Eq. (69) similar to both models [5] and [6].

It is convenient to define the auxiliary value $\sigma_{t r}^{\prime}$ so that

$$
\sigma_{t r}=2 \pi \frac{\xi^{2}}{k^{2}} \sigma_{t r}^{\prime}
$$

Then a nonasymptotic calculation leads to

$$
\sigma_{t r 1,2}^{\prime}=e^{\mp \pi \xi_{1,2}} \int_{0}^{2 k r_{1,2}}\left|U\left(1 \pm i \xi_{1,2}, 1, i z\right)\right|^{2} z d z
$$

with the values

$$
r_{1}=\frac{\left(Z_{1}-K Z_{2}\right)^{1 / 3}}{2 n^{1 / 3}}, \quad r_{2}=\frac{\left(Z_{1}-K Z_{2}\right)^{1 / 3}}{2(n K)^{1 / 3}},
$$

which are defined by one-half of the average distance between donors and acceptors, respectively.
As a result the following expression for carrier mobility can be obtained:

$$
\mu=\frac{2^{5 / 2} \epsilon^{2}\left(k_{B} T\right)^{3 / 2}}{3 \pi^{3 / 2} e^{3} m^{* 1 / 2} n} \int_{0}^{\infty} \frac{x^{3} e^{-x}}{Z_{1}^{2} \sigma_{t r 1}^{\prime}(x)+K Z_{2}^{2} \sigma_{t r 2}^{\prime}(x)} d x .
$$

The integrals over energies can be estimated in a standard way [6]: smoothly changing functions can be taken out of the integrals with the argument $x=3$ when the energy distribution function has its maximum value. This leads to the following analytical expression for the mobility:

$$
\mu=\frac{2^{7 / 2} \epsilon^{2}\left(Z_{1}-K Z_{2}\right)\left(k_{B} T\right)^{3 / 2}}{\pi^{3 / 2} e^{3} m^{* 1 / 2} n\left[Z_{1}^{2} \sigma_{t r 1}^{\prime}\left(3 k_{B} T\right)+K Z_{2}^{2} \sigma_{t r 2}^{\prime}\left(3 k_{B} T\right)\right]} .
$$

If $Z_{1}=Z_{2}=1$ it transforms as follows:

$$
\mu=\frac{2^{7 / 2} \epsilon^{2}(1-K)\left(k_{B} T\right)^{3 / 2}}{\pi^{3 / 2} e^{3} m^{* 1 / 2} n\left[\sigma_{t r 1}^{\prime}\left(3 k_{B} T\right)+K \sigma_{t r 2}^{\prime}\left(3 k_{B} T\right)\right]} .
$$

We can compare this with the analogous formula in the framework of Conwell-Weisskopf model [6]

$$
\mu_{C W}=\frac{2^{7 / 2} \epsilon^{2}(1-K)\left(k_{B} T\right)^{3 / 2}}{\pi^{3 / 2} e^{3} m^{* 1 / 2} n(1+K) \ln \left(1+\left(\frac{3 \epsilon k_{B} T(1-K)^{1 / 3}}{Z e^{2}[n(1+K)]^{1 / 3}}\right)^{2}\right)} .
$$

The results of the calculation using Eqs. (73) and their comparison with Conwell-Weisskopf model results are shown in Fig. 6. The same figure shows that the dependence of the mobility on the temperature and the compensation $K$ in our consideration differs greatly from the results of Conwell-Weisskopf model [6] based on the Rutherford cross section with the phenomenological regularization. In principle, such difference can be revealed in some experiments.

\section{CONCLUSIONS}

In this paper we have studied the question whether the nonintegrable singularity of the Rutherford scattering cross section is an intrinsic feature of the Coulomb problem or one could avoid it following the rigorous rules of the quantum mechanical scattering theory. The reason for this question is in the obvious contradiction between the behavior of the exact wave function of the particle in the Coulomb field

$$
\psi_{\vec{k}}(\vec{r})=e^{ \pm(\pi / 2) \xi} \Gamma(1 \mp i \xi) e^{i \vec{k} \cdot \vec{r}} F[ \pm i \xi, 1, i(k r-\vec{k} \cdot \vec{r})],
$$

and its asymptotic form

$$
\psi_{k}(\vec{r}) \simeq e^{i \vec{k} \cdot \vec{r}}+f(\theta) \frac{e^{i k r}}{r},
$$

which is used in the standard scattering theory.

The former expression is finite within the whole space in contrast to the latter one which tends to infinity along the line $\theta=0$. The answer to the above question is the following: the reason for the singularity in the cross section for a long range Coulomb potential is not physical but is due to an improper mathematical use of the asymptotic representation for the 

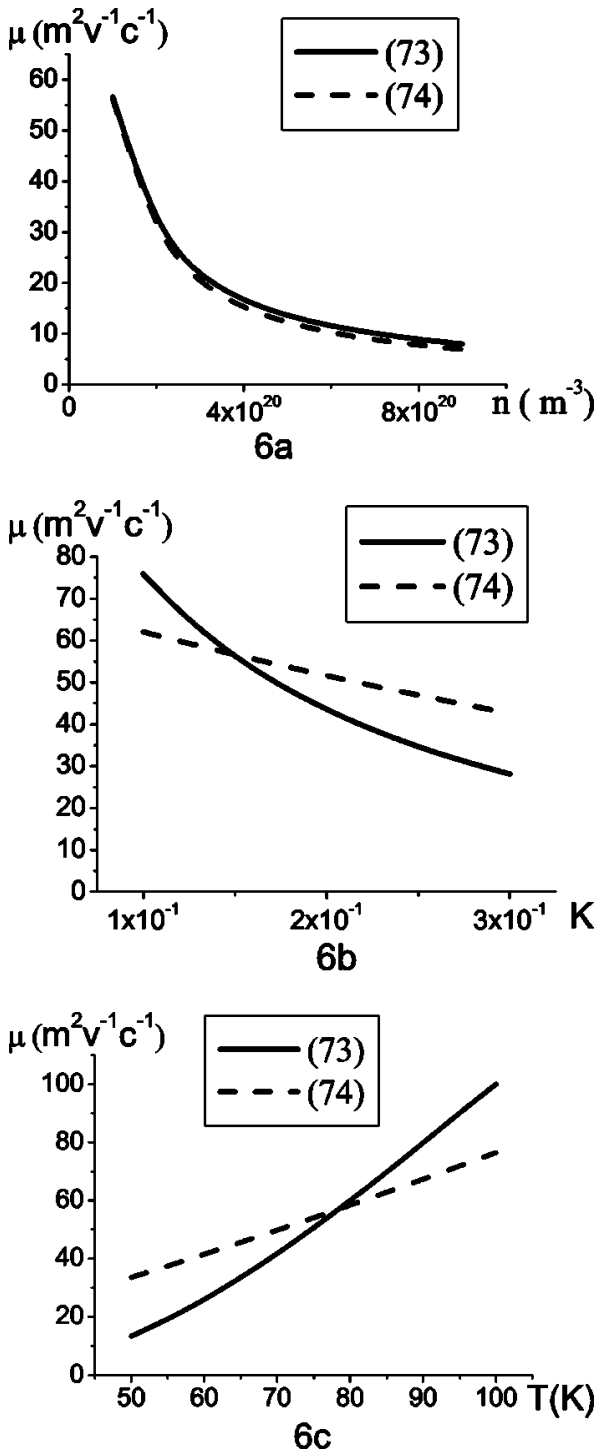

FIG. 6. Comparison of the mobilities calculated with the nonasymptotic transport cross section (solid line) and in the framework of the Conwell-Weisskopf model (dashed line). (a) Dependence on the impurity concentration $n$, (b) on the compensation $K$, and (c) on the temperature $T$. The following parameters are used: $T=78, \epsilon=10$, $m=0.2 m_{0}$, and $K=0.15$.

wave function in the "near" zone which is determined by the cone with the angle $\theta_{0} \simeq \sqrt{2 / k r}$ along $\vec{k}$ (Fig. 1). If one calculates the flux of the scattered particles with the exact wave function, the cross section remains finite in the entire range of angles. This leads to the regularization of the Coulomb scattering problem and permits one to calculate the finite value for the total and transport cross sections and consider the analog of the "optical" theorem for this case. One of the main results of the paper is expressed in formula (28) for the total cross section that is different for attractive and repulsive potentials

$$
\sigma_{\mathrm{tot}}=\frac{2 \pi r}{k} \xi^{2} I_{ \pm}(\xi)
$$

with well-defined functions $I_{ \pm}(\xi)$ of the dimensionless parameter of the Coulomb interaction $\xi=e^{2} / \hbar v$.

The most unusual feature of the regularized cross section is its dependence on the distance $r$ between the detector and the scattering center. The physical meaning of this result becomes clear if one takes into account than in real scattering experiments the flux of incident particles is represented by the wave packet with the transversal width $a$, and the standard interpretation of the scattering data is possible under the condition that the diffraction extension of the wave packet is negligible [13]. This leads to the restriction for the distance $r<k a^{2}$ which is actually fulfilled for real experimental conditions [13]. As a result the maximal value of the total cross section is

$$
\sigma_{\max }=2 \pi a^{2} \xi^{2} I_{ \pm}(\xi) .
$$

It means that the maximal cross section is proportional to the area of the transversal section of the incident particles beam: because of a long range character of the Coulomb potential all particles of the beam are eventually scattered with the probability depending on the parameter $\xi$. So, it is quite natural that when the detector is situated at a smaller distance $r$ from the center, it will register a fewer number of the scattered particles depending on $r$.

It is important to emphasize that the considered peculiarities of the Coulomb problem should be taken into account only for the small angle scattering experiments for the potential without any screening and the processes depending on the integral scattering characteristics. In other cases our results completely coincide with the standard Rutherford consideration.

\section{ACKNOWLEDGMENTS}

The authors are grateful to Professor N. A. Poklonskii for useful discussions and to the International Scientific Technical Center (Grant B-626) for supporting this work.
[1] L. D. Landau and E. M. Lifshitz, Quantum Mechanics: NonRelativistic Theory, 3rd ed. (Pergamon, London, 1997), Vol. 3.

[2] R. G. Newton, Scattering Theory of Waves and Particles, 2nd ed. (McGraw-Hill, New York, 1982).

[3] L. D. Landau and E. M. Lifshitz, Mechanics (Nauka, Moscow, 1965).

[4] J. Synge, Classical Dynamics (Fizmatgiz, Moscow, 1963).
[5] H. Brooks, Phys. Rev. 83, 879 (1951).

[6] E. Conwell and V. F. Weisskopf, Phys. Rev. 77, 388 (1950).

[7] N. A. Poklonskii et al., Appl. Phys. Lett. 93, 9749 (2003).

[8] B. K. Ridley, Quantum Processes in Semiconductors (Clarendon, Oxford, 1999); K. Seeger, Semiconductor Physics (Springer-Verlag, Berlin, 1999).

[9] S. W. Kim, H-K. Park, H-S. Sim, and H. Shomerus, J. Phys. A 
36, 1299 (2003).

[10] K. Elmer, J. Phys. D 34, 3097 (2001).

[11] K. Harigawa, J. Phys.: Condens. Matter 12, 7069 (2000); 12, 388 (2000).

[12] V. G. Baryshevskii, L. N. Korennaya, and I. D. Feranchuk, Sov. Phys. JETP 34, 249 (1972).

[13] M. Goldberger and K. Watson, Collision Theory (Wiley, New York, 1964).

[14] W. Zackowicz, J. Phys. A 36, 4445 (2003).

[15] D. Jackson, Classical Electrodynamics, 2nd ed. (Wiley, New York, 1975).
[16] P. M. Morse and H. Feshbach, Methods of Theoretical Physics (McGraw-Hill, New York, 1953).

[17] J. M. Ziman, Principles of The Theory of Solids (Cambridge University Press, Cambridge, England, 1972).

[18] A. D. Boardman and D. W. Henry, Phys. Status Solidi B 60, 633 (1973).

[19] J. Blakemore, Solid State Physics (Nauka, Moscow, 1988).

[20] E. M. Lifshitz and L. P. Pitaevski, Physical Kinetics (Nauka, Moscow, 1979).

[21] A. F. Nikiforov and V. B. Uvarov, Special Functions of Mathematical Physics (Nauka, Moscow, 1984). 